\title{
Method of error analysis for phase-measuring algorithms applied to photoelasticity
}

\author{
Juan Antonio Quiroga and Agustín González-Cano
}

\begin{abstract}
We present a method of error analysis that can be applied for phase-measuring algorithms applied to photoelasticity. We calculate the contributions to the measurement error of the different elements of a circular polariscope as perturbations of the Jones matrices associated with each element. The Jones matrix of the real polariscope can then be calculated as a sum of the nominal matrix and a series of contributions that depend on the errors associated with each element separately. We apply this method to the analysis of phase-measuring algorithms for the determination of isoclinics and isochromatics, including comparisons with real measurements. (C) 1998 Optical Society of America
\end{abstract}

OCIS codes: $100.2650,120.5050,230.5440,260.5430$.

\section{Introduction}

In recent years phase-measuring algorithms have been widely applied to photoelastic measurements. ${ }^{1-4}$ In every kind of measurement it is of fundamental importance to estimate the errors. An error analysis has two main objectives. First, we must know the accuracy of the measures obtained with our experiment. For this goal a quantitative error analysis (numerical or analytical) is enough. But we might also be interested in an a priori qualitative knowledge of the influence of the different error sources on the final result to design the experimental setup properly or simply to choose the tolerances of the elements to be used.

There exists in the literature a considerable number of papers that deal with the problem of error estimation in phase-measuring algorithms. ${ }^{5,6}$ These papers mainly study the effect in the phase calculation of errors in the phase steps, nonlinearities in the detector, additive noise, etc. When applied to photoelasticity, however, a new error source appears for this type of algorithm, namely, errors associated with the configurations of the polariscope used to obtain the different intensity distributions on which the phase-measuring algorithms are based. The theoretical expressions for

The authors are with the Departamento de Óptica, Facultad de Ciencias Físicas, Universidad Complutense, Ciudad Univeritaria, s/n, 28040 Madrid, Spain.

Received 19 December 1997; revised manuscript received 30 March 1998.

0003-6935/98/204488-08 $\$ 15.00 / 0$

(C) 1998 Optical Society of America the intensity distributions are valid for only definite orientations of the elements of the polariscope. That means that, if we have an error in these orientations, the real output intensity of the polariscope does not coincide with that which is required to obtain the desired phase shifts between intensity distributions, which implies that the calculated phase will be affected by errors. The analytical expressions of these intensity distributions cannot be used as a basis for the analysis of the phase error, since they are true for only the particular configurations of the polariscope for which they are calculated. Therefore the usual method of error propagation cannot be directly applied to this case.

We propose in this paper a different approach to error analysis in phase-measuring algorithms applied to photoelasticity that is based in Jones calculus. The output intensity of the polariscope can be obtained by use of Jones calculus ${ }^{7}$ by the calculation of the matrices corresponding to each element. Then, if we can estimate the errors associated with each element, we can obtain the Jones matrix of the error-affected polariscope and calculate its output intensity. But this direct method is not well suited to qualitative analysis of the contributions of the different error sources. The analytical expressions involved are cumbersome and hard to analyze, and it is difficult to know which is the effect of a determinate contribution on the final error of the phase. General qualitative conclusions are almost impossible to obtain from this kind of analysis.

To be able to perform in an easy way both qualitative and quantitative error analysis, we developed a method based in Jones calculus that uses a first-order 
approximation. Instead of using the exact Jones matrices of the error-affected elements, we associate with each element the Jones matrix of the error-free element plus a perturbation Jones matrix that depends linearly on the error. In this way we can calculate the Jones matrix of the whole polariscope, obtaining the Jones matrix of the error-free polariscope and a series of contributions from the different error sources. This real Jones matrix can then be used to obtain the output intensity of the erroraffected polariscope.

\section{Jones Matrices of Error-Affected Elements}

In Jones calculus an element is depicted by a $2 \times 2$ complex matrix, which can be written as

$$
J=\left[\begin{array}{ll}
j_{11} & j_{12} \\
j_{21} & j_{22}
\end{array}\right] \text {. }
$$

In photoelasticity we are mainly interested in two characteristics of the elements: their orientation with respect to a previously chosen reference system and their retardation when it exists. Let us suppose that the orientation of an element is given by the angle $\theta$. If an orientation error $\Delta \theta$ is present, we can obtain the real Jones matrix of the error-affected element $J^{*}(\theta)$ by applying the general relation

$$
J^{*}(\theta)=J(\theta+\Delta \theta)=S(-\Delta \theta) J(\theta) S(\Delta \theta),
$$

where $S$ is the rotation matrix, given by

$$
S(\beta)=\left[\begin{array}{cc}
\cos \beta & \sin \beta \\
-\sin \beta & \cos \beta
\end{array}\right] .
$$

If we consider $\Delta \theta$ small, we can write approximately

$$
\begin{aligned}
S(\Delta \theta) & =\left[\begin{array}{cc}
1 & \Delta \theta \\
-\Delta \theta & 1
\end{array}\right], \\
J^{*}(\theta) & =\left[\begin{array}{cc}
1 & -\Delta \theta \\
\Delta \theta & 1
\end{array}\right] J(\theta)\left[\begin{array}{cc}
1 & \Delta \theta \\
-\Delta \theta & 1
\end{array}\right] .
\end{aligned}
$$

If we calculate explicitly the elements of $J^{*}(\theta)$ we obtain

$$
\begin{aligned}
{\left[\begin{array}{ll}
j_{11} * & j_{12}^{*} \\
j_{21} * & j_{22}^{*}
\end{array}\right] } & =\left[\begin{array}{ll}
j_{11} & j_{12} \\
j_{21} & j_{22}
\end{array}\right]+\Delta \theta\left[\begin{array}{cc}
-j_{12}-j_{21} & j_{11}-j_{22} \\
j_{11}-j_{22} & j_{12}+j_{21}
\end{array}\right] \\
J^{*}(\theta) & =J(\theta)+\Delta \theta J^{O}(\theta),
\end{aligned}
$$

where

$$
J^{O}(\theta)=\left[\begin{array}{ll}
-j_{12}-j_{21} & j_{11}-j_{22} \\
j_{11}-j_{22} & j_{12}+j_{21}
\end{array}\right]
$$

can be considered as an error matrix because of the bad orientation that is added to the error-free matrix $J(\theta)$.

In the same way we can consider an error in the retardation of a retardation plate. The Jones matrix for a retardation plate of retardation $\eta$ and orienta- tion $\theta$ (angle between the fast axis of the plate and the $x$ axis of the system) is given by

$$
\begin{aligned}
& D(\eta, \theta)= \\
& {\left[\begin{array}{cc}
\exp (i \eta) \cos ^{2} \theta+\sin ^{2} \theta & {[\exp (i \eta)-1] \sin \theta \cos \theta} \\
{[\exp (i \eta)-1] \sin \theta \cos \theta} & \exp (i \eta) \sin ^{2} \theta+\cos ^{2} \theta
\end{array}\right] .}
\end{aligned}
$$

If we assume a small error in the retardation $\Delta \eta$ and no error in the orientation, we have

$$
\begin{aligned}
\exp [i(\eta+\Delta \eta)] & =\exp (i \eta)(1+i \Delta \eta), \\
D^{*}(\eta, \theta) & =D(\eta, \theta)+i \exp (i \eta) \Delta \eta D^{R}(\theta),
\end{aligned}
$$

where $D^{R}(\theta)$ is the error matrix resulting from the retardation and is given by

$$
D^{R}(\theta)=\left[\begin{array}{cc}
\cos ^{2} \theta & \sin \theta \cos \theta \\
\sin \theta \cos \theta & \sin ^{2} \theta
\end{array}\right] .
$$

If we have both retardation and orientation errors in a retardation plate we will have, taking into account only linear contributions on the error,

$$
D^{*}(\eta, \theta)=D(\eta, \theta)+\Delta \theta D^{O}(\eta, \theta)+i \exp (i \eta) \Delta \eta D^{R}(\theta),
$$

where $D^{O}(\eta, \theta)$ can be obtained by the application to Eq. (9) of the general formula of Eq. (8), yielding

$$
\begin{aligned}
& D^{O}(\eta, \theta)= \\
& \quad\left[\begin{array}{cc}
{[1-\exp (i \eta)] \sin 2 \theta} & 1+\exp (i \eta) \cos 2 \theta \\
1+\exp (i \eta) \cos 2 \theta & {[\exp (i \eta)-1] \sin 2 \theta}
\end{array}\right]
\end{aligned}
$$

If we have a polarizer, the only contribution to the error will be the one that is due to orientation:

$$
P^{*}(\theta)=P(\theta)+\Delta \theta P^{O}(\theta),
$$

where $P(\theta)$ is the Jones matrix for a polarizer with the transmission axis at an angle $\theta$ to the chosen $x$ axis, given by

$$
P(\theta)=\left[\begin{array}{cc}
\cos ^{2} \theta & \sin \theta \cos \theta \\
\sin \theta \cos \theta & \sin ^{2} \theta
\end{array}\right],
$$

and $P^{O}(\theta)$ is the corresponding error matrix given by

$$
P^{O}(\theta)=\left[\begin{array}{cc}
-\sin 2 \theta & \cos 2 \theta \\
\cos 2 \theta & \sin 2 \theta
\end{array}\right] .
$$

\section{Output Intensity of the Error-Affected Polariscope}

In experiments of photoelasticity a so-called circular polariscope is usually employed. The light emerging from a polarizer and a quarter-wave plate is incident upon the sample to be studied, which is considered to be a retardation plate with a retardation $\delta$ and an orientation of the fast axis $\alpha$. Both $\delta$ and $\alpha$ depend on a point in the sample. After the sample, a second quarter-wave plate and an analyzer are placed.

We can obtain the Jones matrix of the circular 
polariscope by multiplying the corresponding matrices:

$$
M=P\left(\theta_{4}\right) D\left(\pi / 2, \theta_{3}\right) D(\delta, \alpha) D\left(\pi / 2, \theta_{2}\right) P\left(\theta_{1}\right),
$$

where we used the same notation as in the paragraph above. We now use the formalism introduced in Section 2 to obtain the matrix of the error-affected polariscope in the first-order approximation.

In general, the light beam incident upon the polariscope can be in any state of polarization. To be able to use Jones calculus we must obviate the matrix of the first element $P\left(\theta_{1}\right)$, beginning with its output, as given by

$$
a=\left[\begin{array}{c}
\cos \theta_{1} \\
\sin \theta_{1}
\end{array}\right] .
$$

However, if we consider that the polarizer has a small error of orientation given by $\Delta \theta_{1}$, we can suppose that the beam emerging from the polarizer is given by

$$
a^{*}=\left[\begin{array}{c}
\cos \theta_{1} \\
\sin \theta_{1}
\end{array}\right]+\Delta \theta_{1}\left[\begin{array}{c}
-\sin \theta_{1} \\
\cos \theta_{1}
\end{array}\right]=a+\Delta \theta_{1} a^{o}
$$

Then we have a matrix for the error-affected polariscope, given by

$$
M^{*}=P^{*}\left(\theta_{4}\right) D^{*}\left(\pi / 2, \theta_{3}\right) D(\delta, \alpha) D^{*}\left(\pi / 2, \theta_{2}\right),
$$

where the matrices corresponding to each element can be calculated by Eqs. (13) and (15).

Because we are considering only small errors and we are doing a first-order approximation, we can keep only the terms linear in the errors. We obtain

$$
\begin{aligned}
M^{*}= & M+\Delta \theta_{4} P^{O}\left(\theta_{4}\right) D\left(\pi / 2, \theta_{3}\right) D(\delta, \alpha) D\left(\pi / 2, \theta_{2}\right) \\
& +\Delta \theta_{3} P\left(\theta_{4}\right) D^{O}\left(\pi / 2, \theta_{3}\right) D(\delta, \alpha) D\left(\pi / 2, \theta_{2}\right) \\
& +\Delta \eta_{3} P\left(\theta_{4}\right) D^{R}\left(\pi / 2, \theta_{3}\right) D(\delta, \alpha) D\left(\pi / 2, \theta_{2}\right) \\
& +\Delta \theta_{2} P\left(\theta_{4}\right) D\left(\pi / 2, \theta_{3}\right) D(\delta, \alpha) D^{O}\left(\pi / 2, \theta_{2}\right) \\
& +\Delta \eta_{2} P\left(\theta_{4}\right) D\left(\pi / 2, \theta_{3}\right) D(\delta, \alpha) D^{R}\left(\pi / 2, \theta_{2}\right) .
\end{aligned}
$$

We see that $M^{*}$ is given by the unperturbed matrix $M$ plus the separate contributions of the different errors. Each contribution has the same form: a product of the unperturbed matrices of the elements that are not affected by the error of the perturbed matrix of the element affected by the error. Therefore we can write

$$
M^{*}=M+\sum_{j=1}^{5} \epsilon_{j} E_{j}
$$

where $\epsilon_{j}$ are the errors corresponding to each error source and $E_{j}$ are the products of matrices obtained in the manner depicted above.

Then, if we keep only the terms that are linear in the error, the amplitude of the output beam is given by

$$
b=M^{*} a^{*}=M a+\Delta \theta_{1} M a^{O}+\sum_{j=1}^{5} \epsilon_{j} E_{j} a
$$

and the output intensity is

$$
I^{*}=b^{+} b,
$$

where the superscript plus sign denotes the conjugate transpose. It is easy to see that the output intensity is given by the sum of the error-free output intensity and the contribution of all the error sources. We do

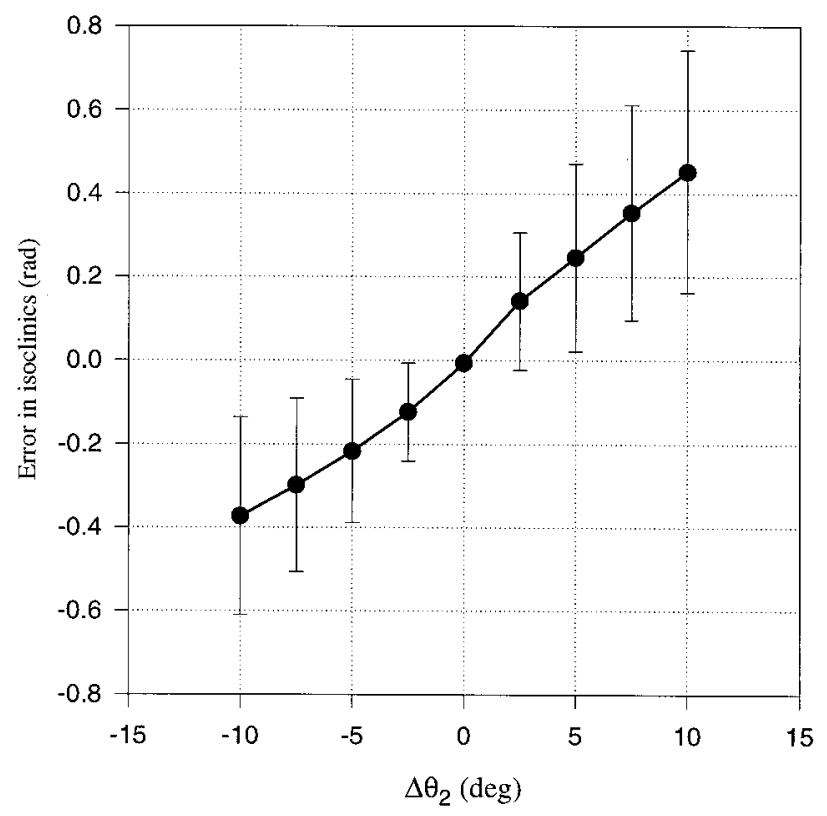

Fig. 1. Evolution of the first moments of the histograms of $\Delta \alpha$ (error in the value of the isoclinic parameter) with $\Delta \theta_{2}$ (error in the orientation of the first quarter-wave plate). The standard deviations of the histograms are plotted as error bars.

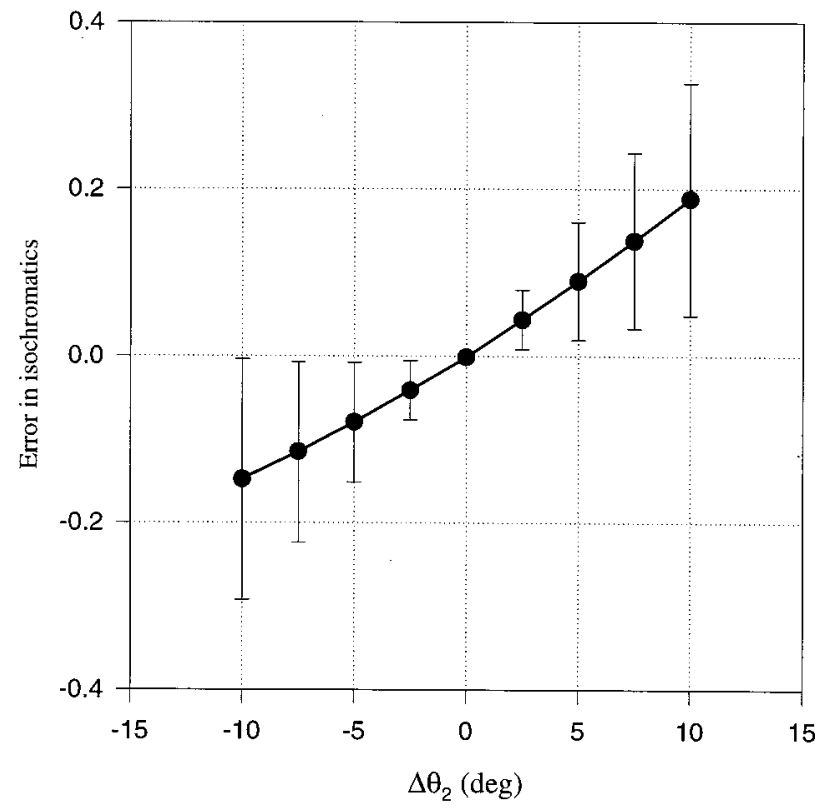

Fig. 2. Evolution of the first moments of the histograms of $\Delta \delta$ (error in the value of the isochromatic parameter) with $\Delta \theta_{2}$ (error in the orientation of the first quarter-wave plate). The standard deviations of the histograms are plotted as error bars. 
(a)

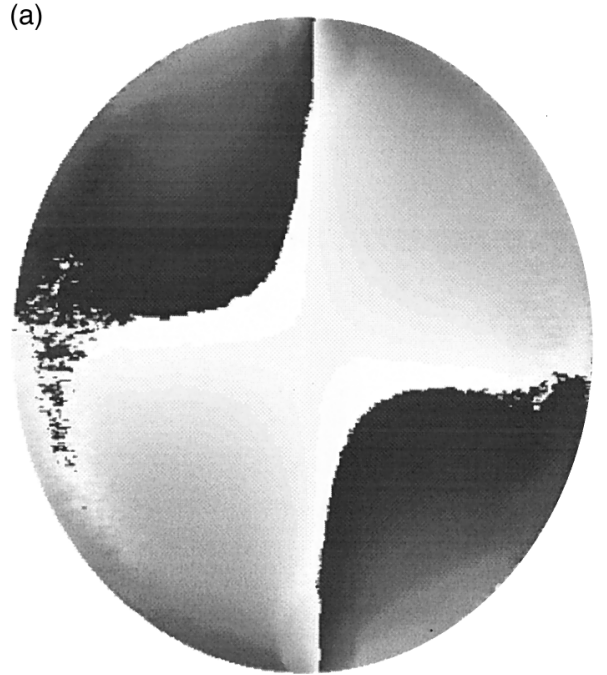

Rad

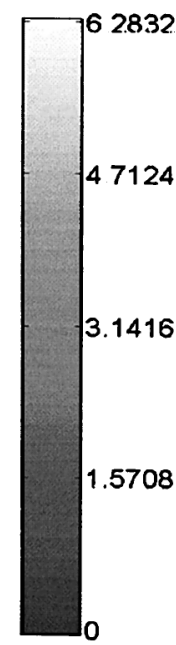

(b)

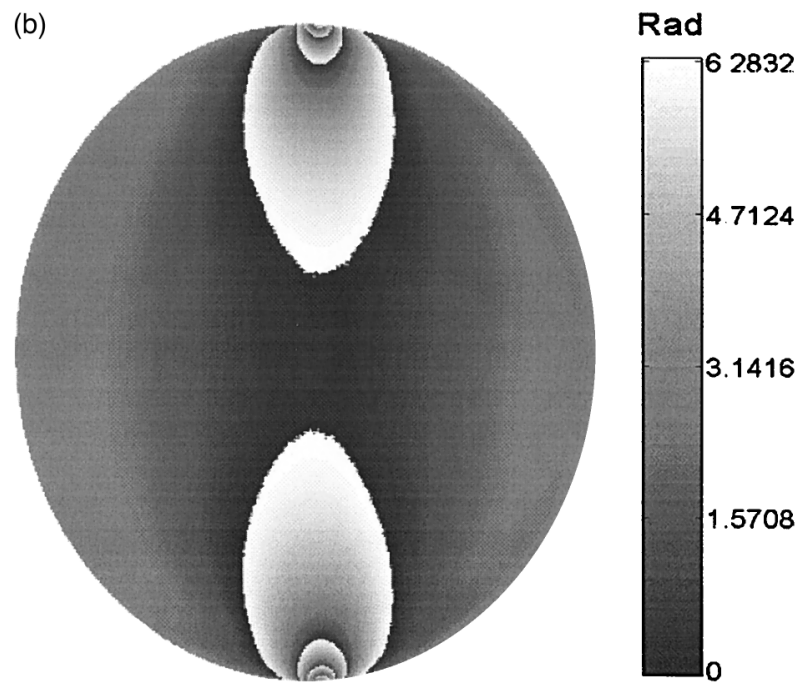

Fig. 3. Distributions of (a) isoclinics and (b) isochromatics in a diametrically loaded disk.

not need to expand Eq. (25) since we use in our calculations Eq. (24) to obtain $b$ and we then simply calculate $I^{*}$ by Eq. (25).

\section{Application to Phase-Measuring Algorithms}

In phase-measuring algorithms a phase is determined as the arctangent of a function of several intensity distributions. In photoelasticity the parameters associated with the sample, $\delta$ and $\alpha$, can be considered as phases. The different intensity distributions correspond to different configurations of the polariscope.

To test the error analysis depicted above, we have chosen two algorithms present in the literature for the determination of isoclinics $\alpha$ (Ref. 2) and isochromatics $\delta .{ }^{4}$ The algorithm for isoclinics is a four-step phase-shift algorithm, and white-light illumination is used to avoid the modulation problems associated with isochromatics. In the case of $\delta$, a modified eight-step algorithm is used with monochromatic light.

The errors in $\alpha$ depend on $\delta$ and vice versa. This
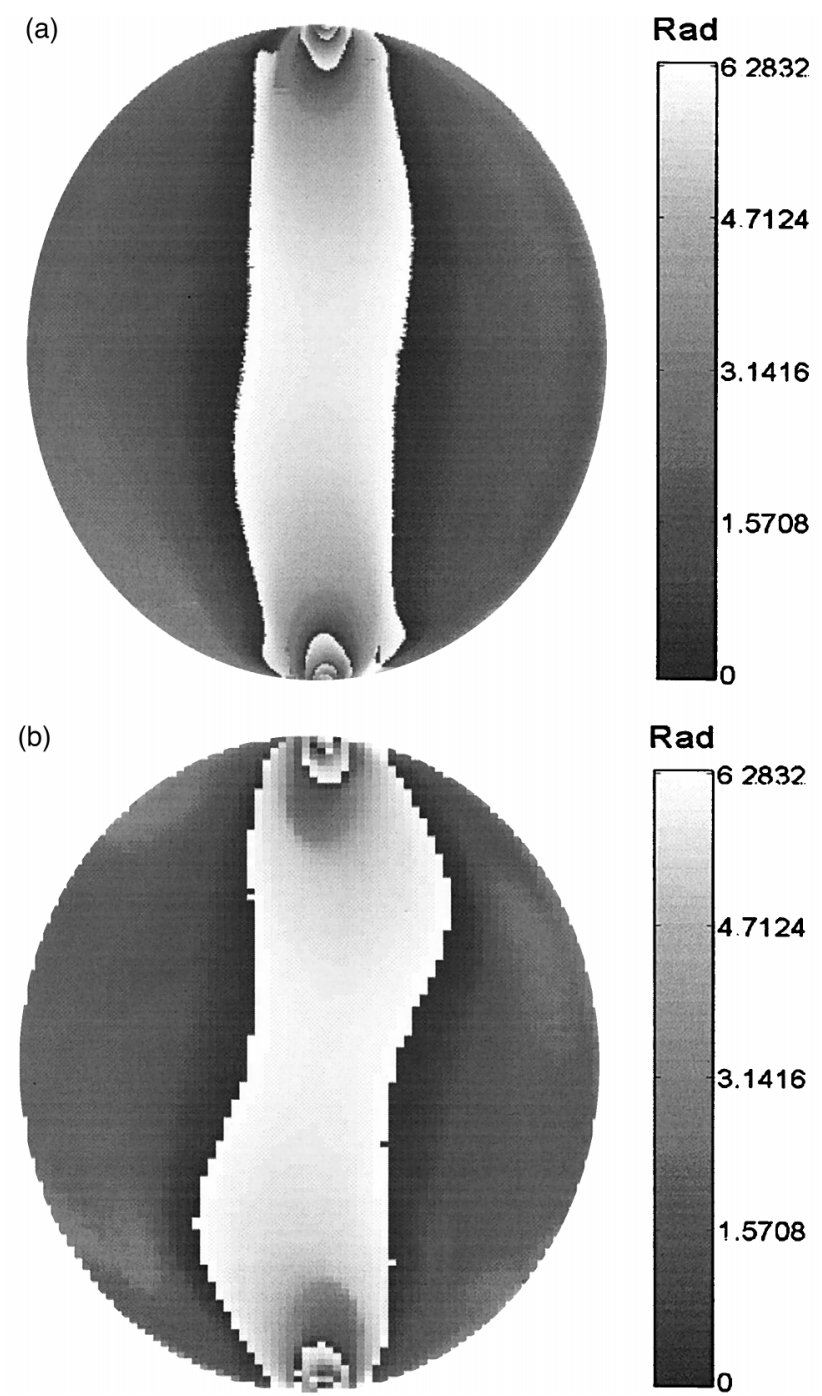

Fig. 4. (a) Experimental values and (b) results of the application of our method obtained for the distribution of isochromatics in a diametrically loaded disk when the following errors are introduced in the polariscope: $+10^{\circ}$ in the orientation of the polarization of the initial beam, $-10^{\circ}$ in the orientation of the first quarter-wave plate, $+10^{\circ}$ in the orientation of the second quarter-wave plate, $-10^{\circ}$ in the orientation of the analyzer.

means that we must find some method that permits us to obtain a certain figure of merit to evaluate the influence of given errors in the polariscope in the final values of the isoclinic and isochromatic parameters $\alpha$ and $\delta$. The method we used can be depicted as follows:

1. We start with known values of $\alpha$ and $\delta$ covering the whole range of variation of these parameters $(0 \leq$ $\alpha \leq \pi / 2 ; 0 \leq \delta \leq 2 \pi$, respectively). In our case, we have used 30 values for each parameter.

2. Taking these values as inputs, we calculate by Eq. (25) the error-affected output intensities of the polariscope with chosen errors in the elements for each of the configurations used by the algorithms. [For instance, we can choose $\Delta \theta_{2}=+5^{\circ}$ and all the other errors equal to zero in Eq. (22).] To simulate 


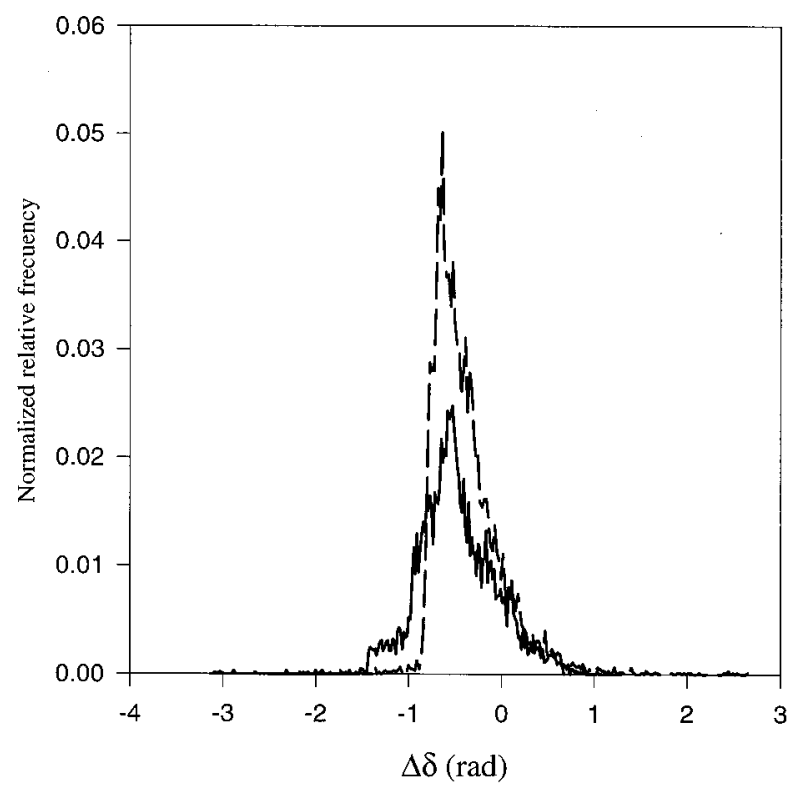

Fig. 5. Histograms of the errors of $\delta$ obtained by our method (solid curve) and by experiment (dashed curve) for the distribution of initial errors of Fig. 4.

white-light illumination in the case of isoclinics, we added the intensities corresponding to 20 different wavelengths from 400 to $700 \mathrm{~nm}$.

3. Using the error-affected intensities, we calculate the corresponding phases $\alpha^{*}$ and $\delta^{*}$. Then we calculate the differences between the error-affected phases and the input values, $\Delta \alpha=\alpha^{*}-\alpha$ and $\Delta \delta=$ $\delta^{*}-\delta$, respectively. We then have two $30 \times 30$ arrays $\Delta \alpha$ and $\Delta \delta$, where the rows correspond to the different input values of $\delta$ and the columns to the values of $\alpha$.

4. Since $\Delta \alpha$ and $\Delta \delta$ depend strongly on $\alpha$ and $\delta$, there exists some dispersion of the values of the elements of the two arrays. We must perform a statistical operation to obtain from this large amount of information one or two figures of merit that permit us to determine the global influence of the chosen initial errors in $\alpha$ and $\delta$. We decided to calculate the histogram of the values of $\Delta \alpha$ and $\Delta \delta$ and analyze them.

5. To obtain numerical estimations of the errors induced in $\alpha$ and $\delta$ by the initial chosen errors, we calculated the first moment and the standard deviation of the histograms, associating the first moment with a systematic error and the standard deviation with a random error.

6. By studying the variations of the first moment and the standard deviation as functions of the initial errors, we can relate the errors in the determination of $\delta$ and $\alpha$ with the different error sources.

For instance, we evaluated the influence of the errors of each element of the polariscope separately. We show as an example the results obtained with initial errors in the orientation of the first quarterwave plate $\Delta \theta_{2}$, from $-10^{\circ}$ to $10^{\circ}$ at intervals of $2.5^{\circ}$. In Figs. 1 and 2 we show the evolution of the first moments of the histograms of $\Delta \alpha$ and $\Delta \delta$ with $\Delta \theta_{2}$. (a)

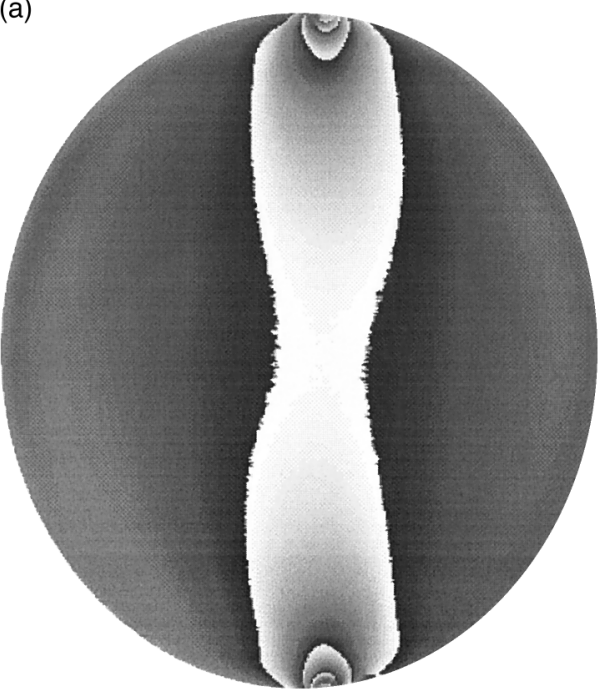

Rad

(b)

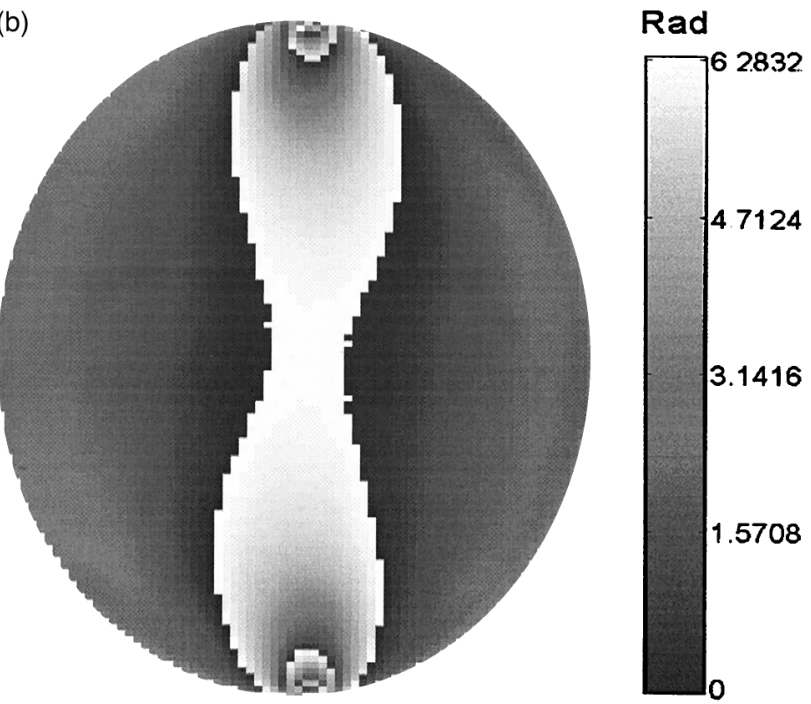

Fig. 6. (a) Experimental values and (b) results of our method obtained for $\delta$ in the disk with initial errors of $+5^{\circ}$ (initial beam), $-5^{\circ}$ (first quarter-wave plate), $+5^{\circ}$ (second quarter-wave plate), and $-5^{\circ}$ (analyzer).

The standard deviations of the histograms are plotted as error bars.

These estimations can be used when the accuracy of the orientations in the experimental setup is known. If the orientation is known to have an error of $\pm 10^{\circ}$, we could expect errors in $\alpha$ of $\pm 0.6 \mathrm{rad}$, whereas the error in $\delta$ could be estimated at $\pm 0.3 \mathrm{rad}$. This kind of information can be useful when designing the experimental setup, and in any case it can be used as an estimate of the accuracy of the measured parameters. In this case it means that errors of $\pm 10^{\circ}$ are not tolerable.

The separate analysis of the contributions of the different elements allow us to make the following conclusions:

1. The errors in $\alpha$ are in general larger than the errors in $\delta$ for the same initial values of errors in the polariscope elements. 


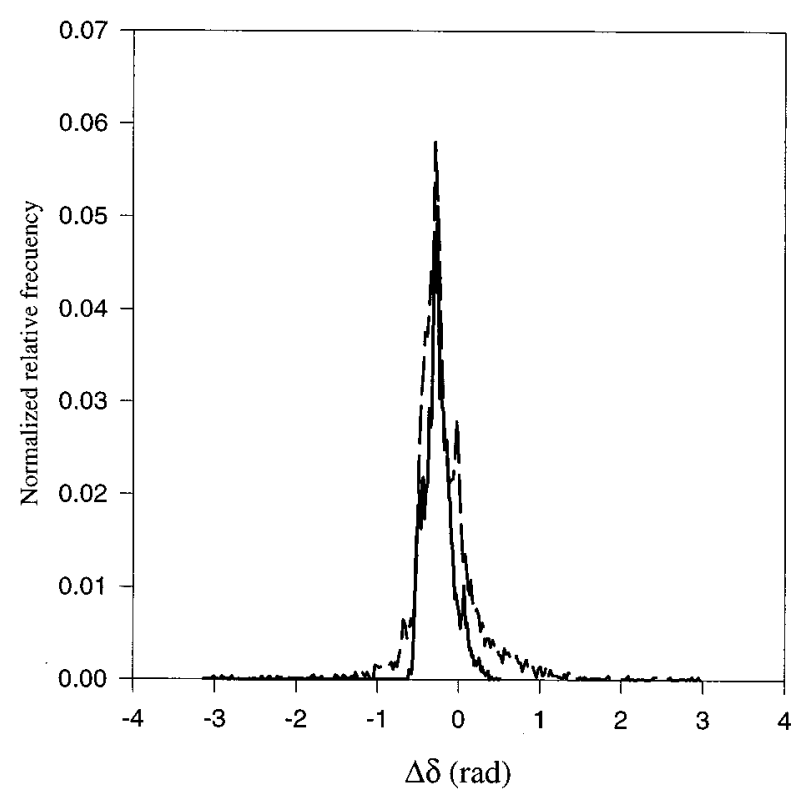

Fig. 7. Histograms of the errors of $\delta$ obtained by our method (solid curve) and by experiment (dashed curve) for the disk with the distribution of initial errors of Fig. 6 .

2. An error in the orientation of the analyzer (if it remains the same in all configurations) does not affect $\delta$. This is also the case with errors in the retardation of the quarter-wave plates and $\alpha$.

3 . The dependence of the error in $\delta$ on the different initial errors of the polariscope elements is quite linear. In the case of $\alpha$ the dependence is also approximately linear, but to a lesser degree. This linearity is not a direct consequence of the linear approximation adopted by us, because this approximation is made with amplitudes and the isochromatics and isoclinics are calculated as nonlinear functions of intensities not amplitudes.
4. The sign of the initial error affects in a definite way the errors in $\alpha$ and $\delta$. In every case the dependence is quite symmetric with respect to the origin.

By use of this method it is immediately possible to estimate the errors that will produce in the measurements of $\delta$ and $\alpha$ any combination of initial errors in the polariscope elements. For instance, if we know the accuracy of the orientations of all the elements, knowledge of the influences of single elements allows us to select the worst combination, and then we can introduce it into the algorithm to estimate the accuracy of the final measurements.

\section{Comparison with Experimental Results}

The depicted application of our method is useful but not completely realistic. In a real sample the distributions of $\alpha$ and $\delta$ may not be regular, and some values may appear more frequently than others. For that reason, we applied the method to the isoclinic and isochromatic distributions obtained by using the polariscope with real samples. Also, this allows us to visualize the effects on the isoclinic and the isochromatic phase maps introduced by several errors in the configurations of the polariscope.

In the first place we used the distributions of isoclinics and isochromatics in the diametrically loaded disk that is shown in Fig. 3. Using the real experimental values obtained for $\alpha$ and $\delta$ (that we suppose to be exact) as input values for our method, we calculate the phase maps that would be obtained with different initial errors in the elements of the polariscope. In this way we can obtain new phase maps that can be compared directly with the error-free ones. By subtracting the error-affected phase maps from the error-free phase maps, we have two matrices, $\Delta \alpha$ and $\Delta \delta$, that can be used in the same way as above. We can then calculate histograms, first moments, etc. (a)

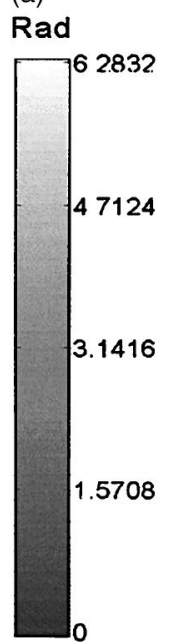

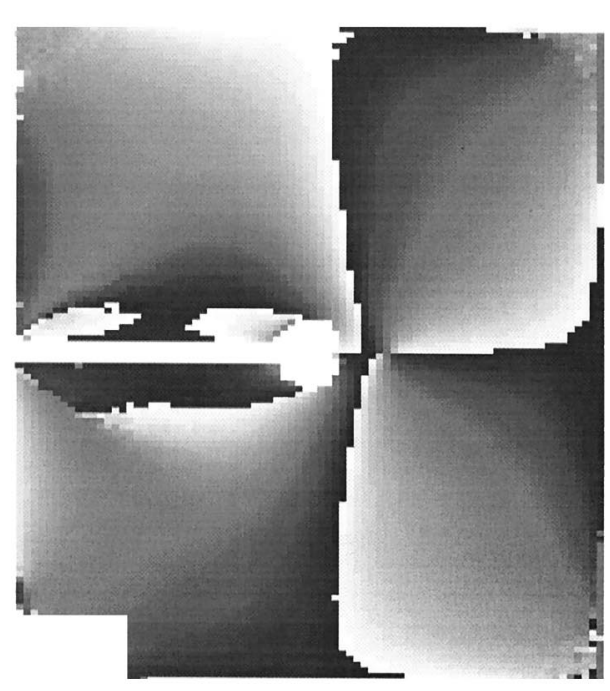

(b)

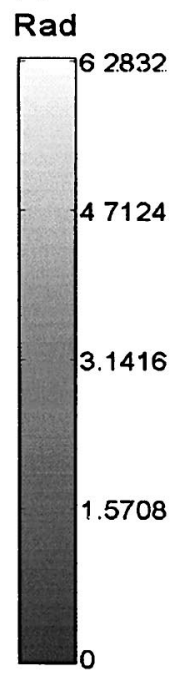

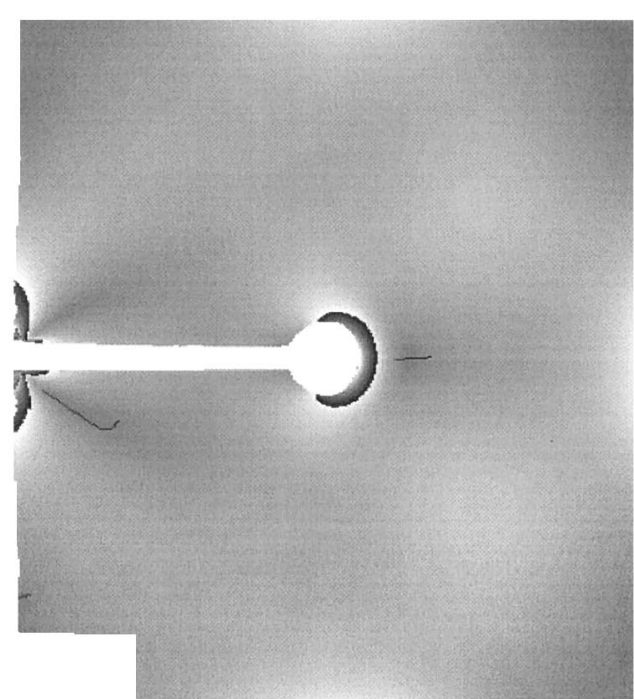

Fig. 8. Distributions of (a) the isoclinics and (b) the isochromatics in a plate with a hole and a cut. 
(a)

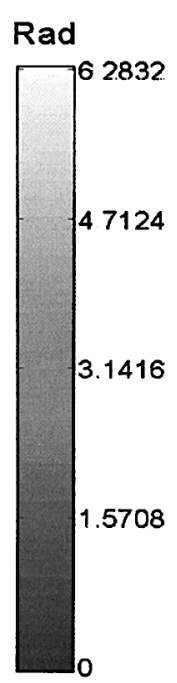

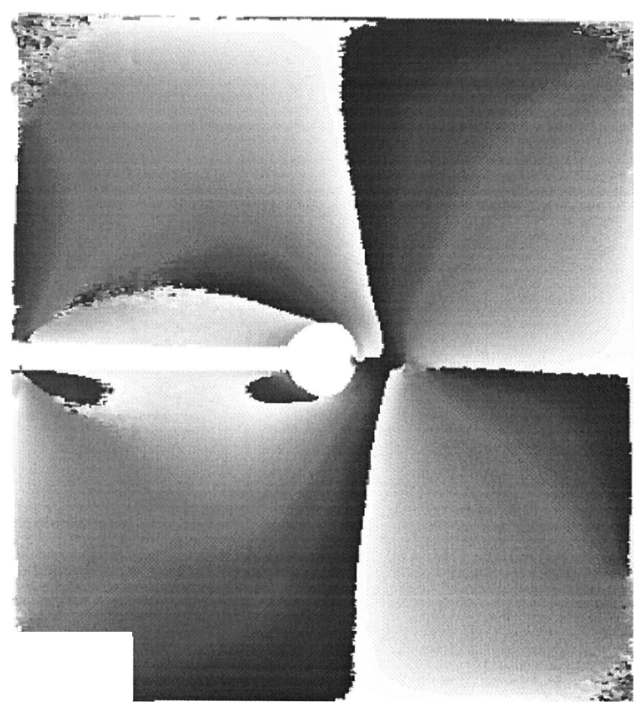

(b)
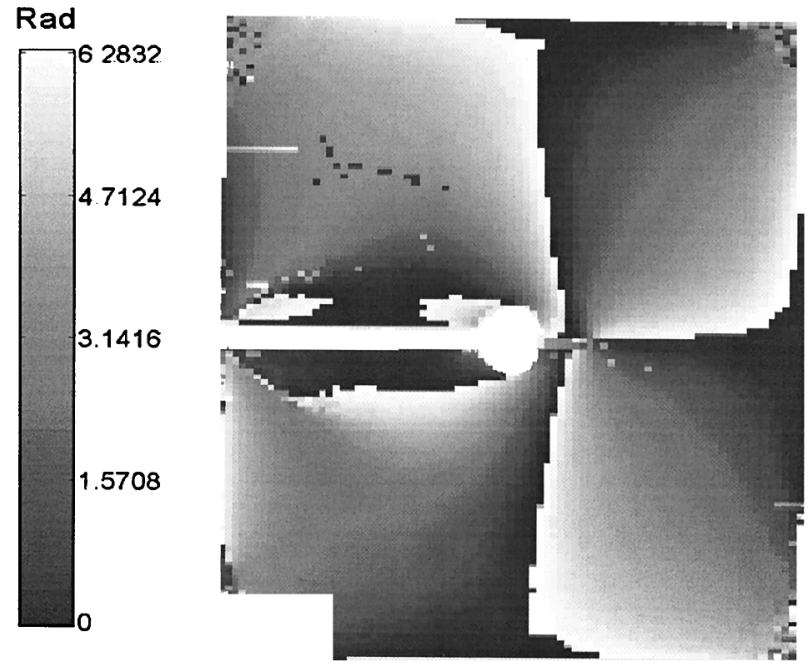

Fig. 9. (a) Experimental values and (b) the results of our method for $\alpha$ in a plate with a distribution of initial errors of $+5^{\circ},-5^{\circ},+5^{\circ}$, and $-5^{\circ}$.

To test the usefulness of the method, we decided to compare it directly with experimental results. For this we obtained the isoclinic and the isochromatic phase maps with some errors introduced into the experimental setup. For instance, the maps shown in Fig. 4 correspond to the isochromatics of the loaded disk when the orientations of the elements of the polariscope have errors of $+10^{\circ}$ (initial beam), $-10^{\circ}$ (first quarter-wave plate), $+10^{\circ}$ (second quarterwave plate), and $-10^{\circ}$ (analyzer). Although these error values might appear to be unrealistically large, we have included this example because it illustrates how the method works when large errors are introduced. In Fig. 4(a) we show the experimental results, and in Fig. 4(b) we show the results of the application of our method with these values for initial errors. In Fig. 5 we show the corresponding histograms. We see that qualitatively the results of the application of our method coincide with the experimental values. Also, there exists good agreement among the numerical values for the positions of maxima (associated with systematic error) and in the width of the distribution (associated with random errors) between experimental and simulated values. In Figs. 6 and 7 we show the results of the application of our method to a distribution of initial errors of $+5^{\circ}$, $-5^{\circ},+5^{\circ}$, and $-5^{\circ}$ in the elements of the polariscope.

Another real sample was used that consisted of a plate with a hole in the center and a cut joining the hole with the border. In Fig. 8 we show the corre- (a)

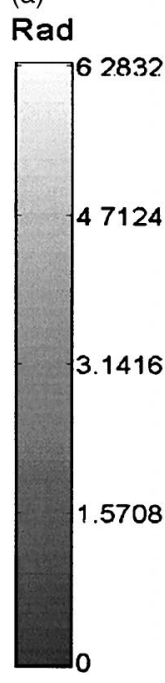

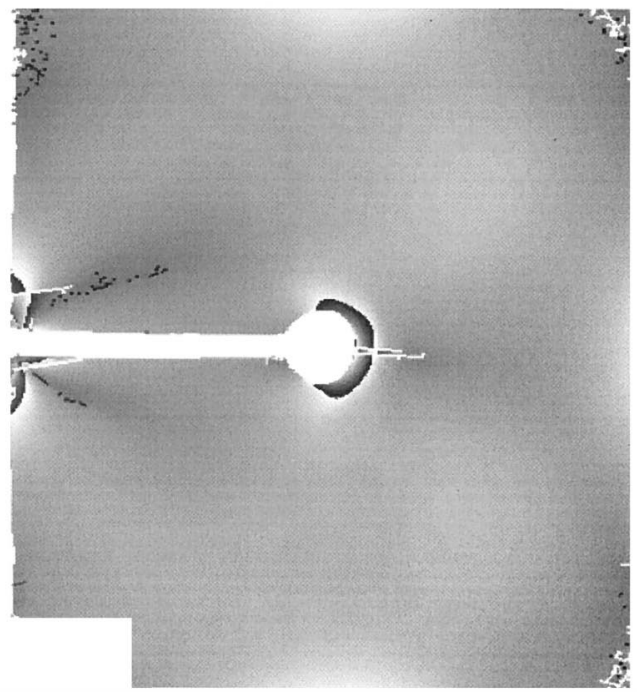

(b)

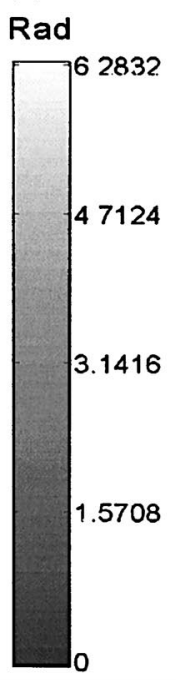

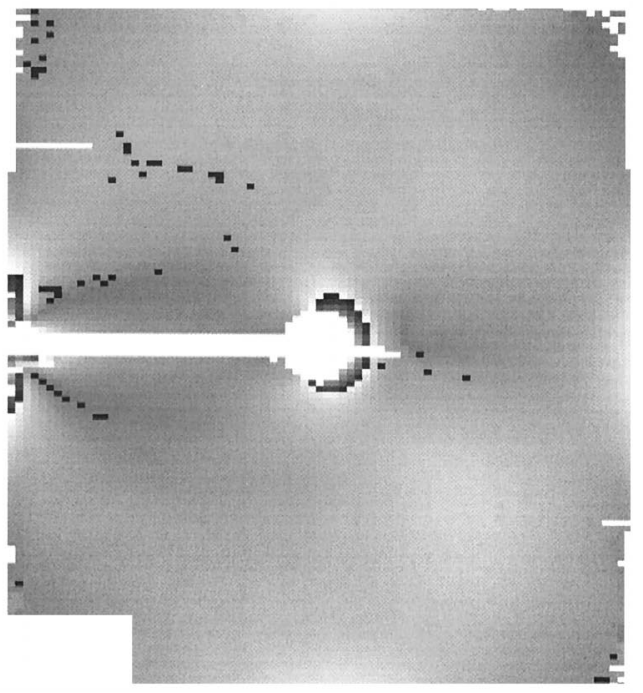

Fig. 10. (a) Experimental values and (b) the results of our method for $\delta$ in a plate with a distribution of initial errors of $+5^{\circ},-5^{\circ},+5^{\circ}$, and $-5^{\circ}$. 


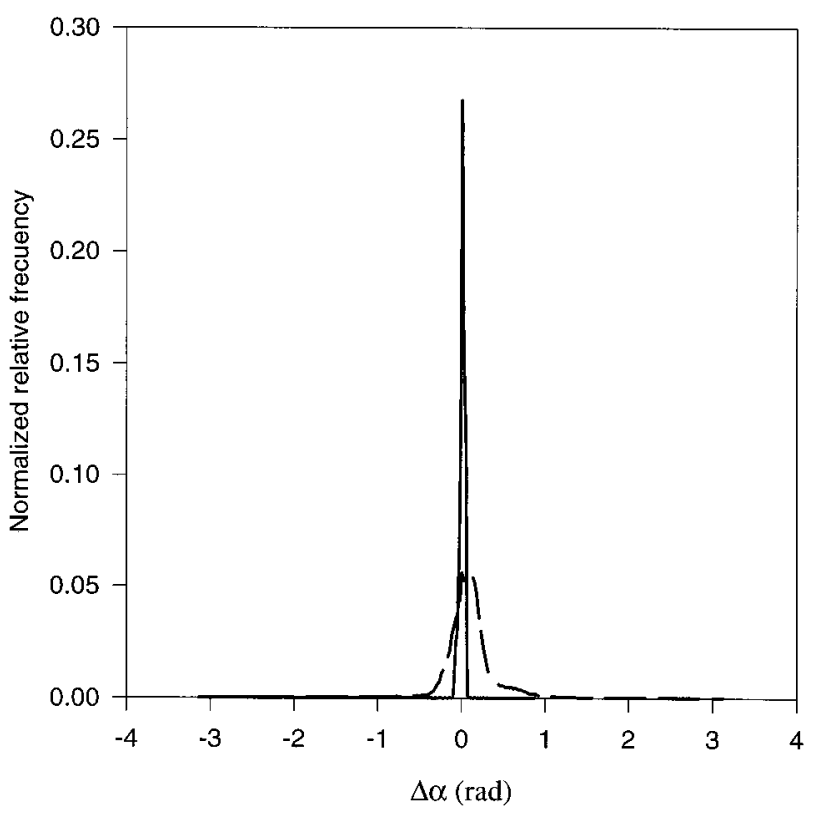

Fig. 11. Histograms of the errors in $\alpha$ obtained with our method (solid curve) and by experiment (dashed curve) for the plate with the distribution of initial errors of Fig. 9.

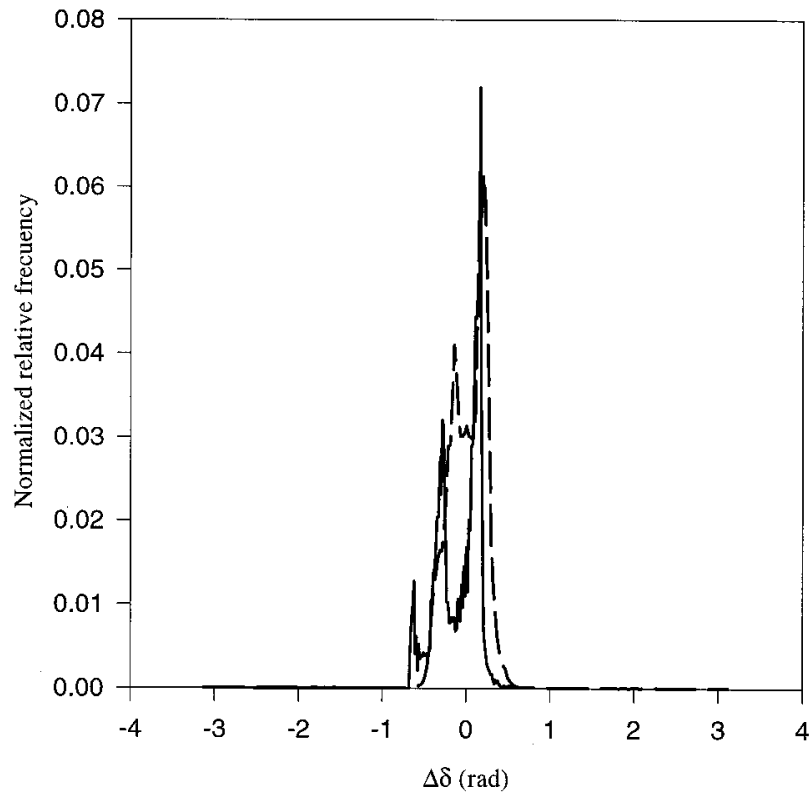

Fig. 12. Histograms of the errors in $\delta$ obtained with our method (solid curve) and by experiment (dashed curve) for the plate with the distribution of initial errors of Fig. 10.

sponding isoclinic and isochromatic distributions. In Fig. 9 we see the experimental values and the results of the application of our method to the isoclinic distribution when the initial errors in the polariscope are $+5^{\circ},-5^{\circ},+5^{\circ}$, and $-5^{\circ}$. Figure 10 shows the same results for the case of isochromatic distributions. Finally, in Figs. 11 and 12 we show the histograms of the errors for the isoclinics and the isochromatics.
It is interesting to note that, in this case, our method can predict the appearance of the histograms of the experimental values as having two peaks. That double-peaked shape is to be expected, as the algorithms include arctangent calculations and the errors associated with the arctangent are typically sinusoidal functions, so different harmonics appear. The agreement between the numerical values for the absolute maxima is good. On the other hand, the two peaks have not been resolved by the algorithm in the case of isoclinics (Fig. 11). However, although the width and the exact shape of the histogram were not predicted in this case by the algorithm, the value of the systematic error can be obtained from it. This shows that our method can be used to estimate the accuracy of the measurements, although in particular cases it is not able to reproduce the details of the behavior of the errors.

\section{Conclusions}

We have depicted a method of error analysis in the application of phase-measuring algorithms to experiments of photoelasticity that is based in Jones calculus. It is conceptually simple and well suited for numerical calculation. We have tested it by both simulation and experiment. It can be used to estimate the contributions of the different error sources to the inaccuracy in the measurements of the isoclinic and the isochromatic parameters. The agreement between simulated and experimental values is good.

We thank Eusebio Bernabeu, Director of the Optics Department of the Universidad Complutense, for his help and continual support. Also, we wish to thank Hans Steinbichler, Steinbichler Optotechnik, GmbH, for helping us with the acquisition of experimental images. This work was partially supported by project MAT 95-0767-C02-02 of the Comisión Interministerial de Ciencia y Tecnología of Spain.

\section{References}

1. C. Buckberry and D. Towers, "Automatic analysis of isochromatic and isoclinic fringes in photoelasticity using phasemeasuring techniques," Meas. Sci. Technol. 6, 1227-1235 (1995).

2. T. Franz, A. Maidhof, and J. Sun, "Verfahren und Vorrichtung zur Bestimmung der Isochromatenwerte in der Spannungsoptik," German patent DE-195 03851 A1 (10 August 1995).

3. A. D. Nurse, "Full-field automated photoelasticity by use of a three-wavelength approach to phase stepping," Appl. Opt. 36, 5781-5786 (1997).

4. J. A. Quiroga and A. González-Cano, "Phase-measuring algorithm for the extraction of isochromatics of photoelastic fringe patterns," Appl. Opt. 36, 8397-8402 (1997).

5. K. Freischlad and C. L. Kouliopoulos, "Fourier description of digital phase-measuring interferometry," J. Opt. Soc. Am A 7, 542-551 (1990).

6. J. van Wingerden, H. J. Frankena, and C. Smorenburg, "Linear approximation for measurement errors in phase-shifting interferometry," Appl. Opt. 30, 2718-2729 (1991).

7. P. S. Theocaris and E. E. Gdoutos, Matrix Methods in Photoelasticity (Springer-Verlag, Berlin, 1979). 